\title{
The Influence of Cultural Preferences on User Interface Design - Polish Case Study
}

\author{
Adam Wojciechowski, Danuta Zakrzewska \\ Institute of Computer Science, Technical University of Lodz, \\ Wolczanska 215, 93-005 Lodz, Poland
}

\begin{abstract}
Sustainability of international virtual learning communities often appears to be problematic. There are many factors influencing it. The paper deals with the influence of cultural preferences on user interface designing process, which in turn may affect information accessibility and data interaction process - the elements of extended TAM model [1]. We consider Polish users' preferences and needs towards user interface appearance. Paper answers the question if there exist Polish cultural preferences and how they may affect user interface designing. The research is based on an existing Socrates Minerva funded CAB project virtual learning community.
\end{abstract}

\section{INTRODUCTION}

Recently, a big amount of international virtual learning communities appeared in the Web. At the beginning they attract the members but sustainability of them is very often problematic. Acceptance of the technology usually plays a big role in it. In [1], there was introduced extended TAM (Technology Acceptance Model)- the model containing all the factors that may influence sustainability of virtual learning communities. Teo, Chan, Wei \& Zhang showed, in their paper, that both information accessibility and community adaptivity have significant effects on user perceptions and behavioural intention to use the system. They indicated that information content and amount, access policies, the type of communication channels provided and information organisation, all have an influence on virtual communities. They emphasized the significance of user adaptation ability as one of critical success factors.

Information accessibility is usually connected with functionality and usability of the software and is determined by its ease of use and especially intuitiveness. The key role in co-operation between the human beings and the system plays user interface. Brenda Laurel says that interface is the contact surface of the thing [2]. However in case of computers, user interface can be treated as a place where user has contact with the software. This place should fulfil user's communication and information needs.

People from different cultures (countries) use interface in a different way, anticipate different graphical layouts, have different expectations and patterns of behaviour, use different metaphors. Therefore, the user interface designers should take

The work was supported by Socrates Minerva CAB project 110681-CP-12003-1-UK-MINERVA-M. into consideration the users' needs and anticipations that arise from culture preferences.

In the paper we present the research that was done on the cultural preferences of Polish students belonging into CAB (Collaboration Across Borders) international virtual learning community. After short description of relevant research, we present the research method, based on the specially prepared questionnaire. In the next sections, we analyse the obtained results and finally give some conclusions and advices for user interface designers.

\section{ReleVANT Research}

In last years many authors examined the factors that may influence user's perception of the interface. According to Raskin [3] successful user interface design is based on considering both ergonomic and cognetic features. Traditionally, design is subdivided into three macrocategories, based on meaningful characteristics [4]:

- information representation and appearance,

- access-navigation-orientation,

- informative content architecture.

Many authors [2,5,6,7,8] have distinguished five main elements of the interface connected with specific user requirements:

- metaphors,

- mental model,

- navigation,

- interaction,

- appearance.

In the previous research, concerning culture preferences, the focus was made on the role of technical features, in adaptation of user interfaces to specified culture, taking into account such factors as appropriate currencies, measurement systems and the language solutions like translation, adapted terminology and using of idiomatic expressions.

But perfect interface should meet also individual user preferences. Unfortunately, it is very difficult to achieve and such adjustable products are not available so far. Numerous software companies usually provide software with interface affected by programmers bias. The problem, which is not big in case of the local market becomes more significant, while the software is sold globally for users representing different nationalities and different cultures.

Due to users' different localisation, there is a need of interface diversification. To make it thoroughly, all aspects of 
user interface appearance and functionality should be taken into consideration. Our previous work [9] was concentrated on the detailed analysis of cultural aspects affecting user interface design with the focus on cultural dimensions. The last ones were also thoroughly described by Baumgartner [2], Trompenaars [10], Condon [11], Victor [12], Hall [13], Hofstede [14] or Marcus [15]. Each of the authors has concentrated on different cultural aspects but merely the whole set of dimensions is the ideal background for national preferences survey [9]. Since Polish user cultural profile has not been examined yet, this paper aims to retrieve prevailing tendencies in polish people's behaviour and their preferences concerning user interface design.

\section{RESEARCH METHOD}

While examining the sustainability of CAB project community, several tools connected with different factors influencing it, were created. There were considered groups of methods connected with such aspects as social and pedagogical benefits and acceptance of the technology. The influence of the culture preferences on the last element was one of the goal of the examination.

As the main research tool, we used the questionnaire comprising 42 questions quantifying cultural dimensions' importance and theirs influence on user interface design. Questions were posed in polish language to avoid any misunderstandings. Answering consisted in the choice between strong confirmation or rejection of a certain cultural behaviour, as well as rather confirmation or rather rejection of it or staying neutral. However, at this stage of the research, in the final analysis we didn't differentiate between strong and normal confirmation of the cultural dimension or its strong or normal rejection.

The research was done on the basis of the answers of Computer Science students of the Technical University of Lodz. The amount of 65 students volunteers attending Multimedia Systems classes took part in the survey. All the participants had a basic knowledge about user interface designing.

Questions were divided into four groups, each of which concerning different problems. Some examples of them are presented in the Table I.

The first set of questions was to reveal students attitude towards achievements and their personal activity. Some of the questions concerned time perception, human adaptability and creativity. Some of them were to show how openly feelings should be expressed and how much information people retrieve from the context.

The second group of the questions concerned polish political and economical situation on an international market. Questions were to show authority conception, the culture strength in international comparison and international co-operation. Some questions in this group were to reveal polish economic progress and political decentralisation.

The third group of questions dealt with polish psychological profile. Their goal was to show polish national
TABLE I

EXEMPLARY QUESTIONS

\begin{tabular}{|l|l|}
\hline \multicolumn{1}{|c|}{ Group } & \multicolumn{1}{c|}{ Questions } \\
\hline $\begin{array}{l}\text { I -Attitude towards } \\
\text { achievements }\end{array}$ & $\begin{array}{l}\text { Do you feel that Polish appreciate achievements } \\
\text { more than properties? } \\
\text { Do you think that emotions should be hidden while } \\
\text { acting? } \\
\text { Do you find Polish flexible and creative? }\end{array}$ \\
\hline $\begin{array}{l}\text { II - Polish culture on } \\
\text { international market }\end{array}$ & $\begin{array}{l}\text { Do you find that Polish prefer democratic } \\
\text { government rather than authoritative ? } \\
\text { Do you feel that Poland have the influence on the } \\
\text { international situation? } \\
\text { Do you think that it is allowed to enter the public } \\
\text { places without asking the permission? }\end{array}$ \\
\hline $\begin{array}{l}\text { III - Polish } \\
\text { psychological profile }\end{array}$ & $\begin{array}{l}\text { Do Polish people like working in teams? } \\
\text { Are Polish traditionalists ? } \\
\text { Do Polish appreciate money and properties? } \\
\text { Do you think that human relations should be stiff } \\
\text { and depend on positions? }\end{array}$ \\
\hline $\begin{array}{l}\text { IV - Polish , } \\
\text { technology and } \\
\text { intellectual property }\end{array}$ & $\begin{array}{l}\text { Do Polish appreciate the role of the technology? } \\
\text { Do you find Polish as open minded? } \\
\text { Do you find Polish culture is based on the } \\
\text { technology ? } \\
\text { Are Polish legalists? }\end{array}$ \\
\hline
\end{tabular}

features like: enthusiasm, hopefulness, pessimism, facesaving ability, perseverance, thrift and spiritual or material goods preferences. Additional goal of the survey was to present polish internal and external relationships. The features comprised: gender roles, subordinate distance, instrumentalism, individualism and collectivism.

Final set of questions were to estimate polish harmonisation with nature, technical development rate, uncertainty accompanying new technologies and intellectual property perception.

The main goal of the answers' analysis was to reveal polish cultural dimensions spectrum [9] and to determine what should be modified in actually used polish user interfaces not appearance deteriorating and intuitiveness declining at the same time. The results of the survey answered the question how different polish user interface might look from actually used patterns and the significance of the investigation of the culture preferences on user interface designing.

\section{ANALYSIS OF THE RESULTS}

\section{A. Attitude towards Achievements}

First group of questions was to show how people perceive their achievements and to which extend they ascribe position to titles of nobility [10]. More then a half (54\%) of respondents stated that Polish are achievement oriented rather than ascriptive oriented. Almost 40\% responded contrarily. Students claim that level of pessimism increases with age of respondents. It means that for young people it is still more important what someone DOES rather than what someone IS. These factors can become important when using titles. For example in ascriptive countries address forms would have the need to provide fields for entering titles of nobility. In case of ascriptive virtual communities it becomes a critical element as the web is a medium that allows for a new status achievement. 
High level of activity orientation [11,16] is confirmed by responses to a subsequent question. Nearly $73 \%$ of questioned students declared themselves as doing-oriented rather then being-oriented. They claimed to like action, efficiency and to concentrate on things that can be observed or measured. Most of them were unwilling to take time to discuss first and understand well complex things. Activity orientation can play a big role when designing metaphors and interaction process of the interface. Active people prefer working metaphors that show manual work rather than brainwork. Polish activity goes with their polichronic time attention [10,12,13]. People used to do several things at one time and their plans used to be constantly shifted (69\%). Persons representing such an attitude prefer browsing through the information space rather than writing precise queries for information retrieval. Yet this factor seems to be age dependant it really suggests how linearly or serendipitously materials should be presented.

Users' poll revealed that over $85 \%$ of persons believe that feelings should be expressed openly, reactions might be shown immediately by means of verbal, mimic or body communications (affective culture [10]). Only 12\% of students believe that emotions confuse the issues and that is why they should not be expressed and shown to the public (neutral culture). This dimension affect user interface interactive feedback. It might be adjusted to the user profile. Discussion with neutralists should focus on the object or proposition while discussion with affectivists may be enthusiastic and vehement and need not to concentrate on subject but on persons involved too.

Reasonably great amount of questioned persons (66\%) presented themselves as low contextual, what means that they prefer information stated clearly but not derived much from context $[12,13]$. A low context communication is similar to communication with a computer - not explicit information causes programme distortions. This factor has an impact on all aspects of interface design. Clear understanding of the interface context is critical for having a successful or positive experience. Users may benefit from high-context graphic only in cultures where high-context communication is common and can be understood. High-context orientation lets interface

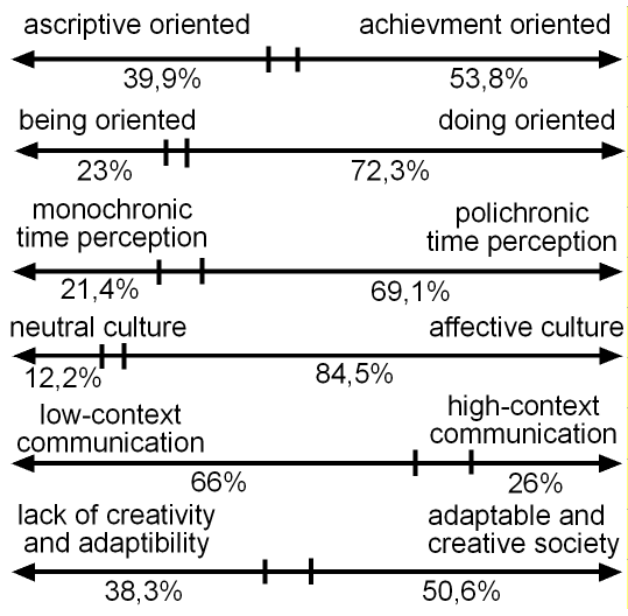

Fig.1. The results of the first set of questions designers support navigation and interaction process more efficiently. The exact results of the first set questions analysis is presented on Fig.1.

Polish level of creativity and new situations' adaptability was presented ambiguously [17]. Almost 50\% of students described themselves as creative and adaptable while above $40 \%$ of questioned users expressed their lack of flexibility and adaptability. It should be emphasized that survey was carried out among students and probably adults' opinion would be different (even less of creative and flexible representatives) . The results may affect not only user interface design but economic progress as well. They show that polish prefer already known solutions and are rather unwilling to new concepts. This might be the hangover from the previous communist system. It means that new interface design solutions become more and more popular as the need for experimenting increases.

\section{B. Authority Conception}

Authority conception ranges from democratic, through authority-centred to authoritarian $[10,12]$. Polish respondents opted generally (77\%) for democracy. This factor influences users' mental model and interaction methods. Society brought up according to democratic rules claims to have rights to question authority contrarily to authoritarian societies. Such dimensions may influence users' reactions and their involvement in the discussion. Uncertainty of discussion moderator existence or uncertainty of presented materials may determine tone of words, language style and freedom of thoughts expression.

Questions concerning degree of strength of a country/culture in international comparison [17] revealed that polish have the complex of minority. Almost 90\% of respondents stated that they have minor position on international market. This feature has shown that Polish are soaked up with western Europe and American patterns. Even people having own point of view different form prevailing solutions hesitate to present it and only accept abroad tendencies. This is mainly the problem of eastern Europe countries and it concerns also Poland. Nevertheless the considerable improvement of Polish international position or increasing of national individualism may cause rapid need for polish interface construction and may speed up process of polish requirements expression.

Political decentralisation dimension [17] pertains believes that decisions made with participation of greater amount of people would be more relevant to diverse interests of society then those made only by political authorities. $49 \%$ of respondents present lethargy and indifferent attitude whereas only $40 \%$ represent enthusiastic and full of energy point of view. Lack of hopefulness was revealed in answers regarding power of distance perception [14]. Very scarcely less powerful members of institutions and organisations accept that power is distributed equally. Huge unemployment level and intimidation, that take place in Poland, cause that relation between boss and subordinate is strictly ruled and dependant on the decisions of the boss (68\%). Only several 
institutions governed in a modern manner tend to diminish distance between bosses and subordinates, that try to work closely together and consult with each other. These observations are verified by the dimension of space [13] perceiving. It refers to the invisible boundary around the individual that is considered as "personal". 74\% claim that they would not accept entering a public space (office) without prior permission. That culture dimension may affect fields of "feedback mechanisms", "open/restricted access", "error messages" and the "use of colours". It may also influence sharing of a workstation (i.e. individual vs. group access), providing personal information (registration dialogues) or interfaces in public space. Overwhelming level of pessimism explains why Polish are not eager to express their own feelings and requirements. The fact that low power distance model become more and more popular is consoling and it means that this research is very important. All the results of the second part of the survey are shown on Fig.2.

\section{Psychological Profile}

Polish perceive themselves as fast moving market where plans are made in short-term perspective. We stand for fostering of virtues related to the past or present. Even though most of us are traditionalists (65\%) we do not bother with fulfilling social obligations and 'face' preservation (only 33\% respect it). We are go-getters, yet we do not pay attention to thrift and we are not persevering (71\%). Most of Polish strive for accumulation of money and other goods (87\%) while spiritual maturity is important only for $13 \%$ of respondents. Such an economical and material bias may influence regarding feedback like visualisation of progress bars and progress in general. It influences the amount of efforts user is willing to make in order to get to know the product, and to use it. We seems to be rather impatient and our interests are benefits-oriented.

Polish students have appeared not to put attention to facesaving. Almost 57\% of them stated that they would be able to loose their prestige and outward dignity for the sake of

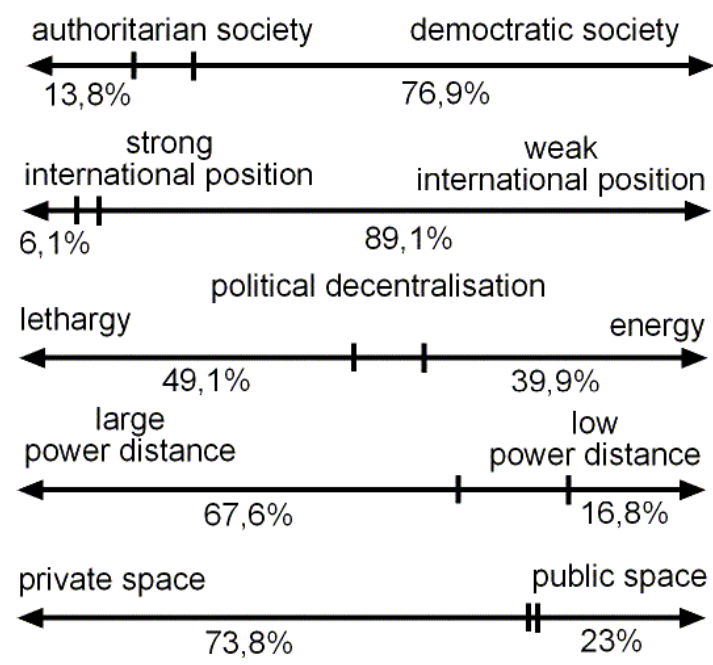

Fig.2. The results of the second part of the survey negotiation deal. Only 11\% definitely stated that face saving is more important then business deals. This society feature affects mental model and interaction process carried out by means of the interface. Respecting considerable differences in this field among certain countries user interface should provide descent business transaction security. This might be critical for interface acceptance and having confidence in it for commerce and social transactions.

When describing gender roles, results were divided into two equal groups [14]. Almost half (47\%) of people state that social gender roles are clearly distinct while the second half (48\%) claim that gender roles overlap. This reveals considerable change in young people point of view, because traditional polish pattern ascribe to both genders explicit roles (men are supposed to be assertive, tough, and focused on material success, whereas women are supposed to be more modest, tender, and concerned with the quality of life). Unquestionable is the fact that significant gender differences exist, both cultural and biological. Besides public state that default design typically favours the male preferences, both gender's preferences become to be considered. Even though systems have no expectation of masculinity or femininity, calls to action (sale conversation) may require direct appeal to gender role.

Almost each of questionnaire respondents have revealed its nature as basically good (90\%). At the same time most of people (70\%) declared themselves as being able to change. Essential is the fact that according to human nature orientation, people need to trust the content they read. This dimension shows how a big effort has to be made for gaining users' trust. Polish are generally confidential until the moment when something abuse their trust. On the other hand accomplishing anything should affect in changes. From this point of view Polish pertain to a group of adjustable persons. That is why new, wisely introduced, user interface concepts may meet users acceptance.

Polish have appeared to be individualists (77\%) with still growing work-in-group abilities (37\%) [10,11,14,16,18]. Whereas about $51 \%$ of respondents denied their will for working collectively with other people (see Fig. 3). It means that Polish look after themselves and their family and do not feel integrated into strong cohesive groups. This cultural dimension may affect user interface design when co-operating by means of electronic media. User interface, especially those in groupware category, must be able to deal with varying levels of individuality and anonymity that are required [19]. Culture in which people tend to value relationships for what they can do for them rather than the relationship itself is called instrumental [18]. Polish students revealed such tendency (53\%) stating that most relationships have economic bias. Only $38 \%$ of respondents showed that pure friendships does really exist. This factor is important especially in establishing a relationship between the interface and the user or group of users, i.e. in a web site striving to attract and retain a certain group of visitors. Society instrumentalism especially may have a great impact on virtual communities 


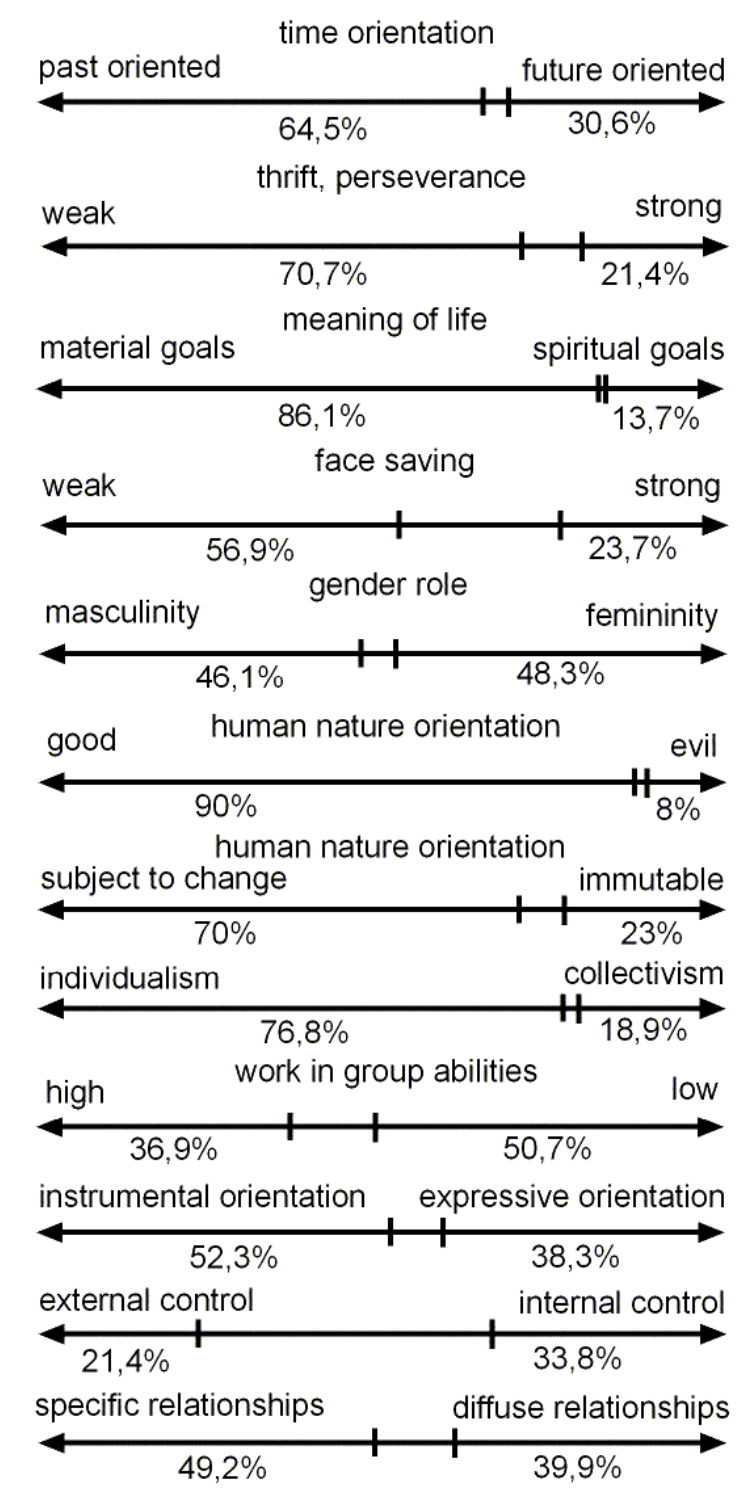

Fig. 3. Results of the third part of the survey.

sustainability. They can mainly survive due to unselfish and honest community participation. People with instrumental attitude perceive virtual co-operation as worthless and lost of time.

Merely $21 \%$ of people perceive nature as dominating and more powerful then the individual. Such attitude can be called externalism $[2,10,16]$ and it proclaims harmonisation with nature and its emulation in technique issues. 34\% of inquired declared themselves as internalists and see the major force in life, its virtue which resides within the individual. The great amount of people hesitated to confirm their adhesion. This social dimension is crucial for interface design process in order to forecast possible users' reaction and technology acceptance. Internalists have tendencies to blame themselves if they can not use the system, since they are not adapted to the technology yet. Externalists would rather blame the system designer, since they require technical issues to be dominated by people. Individualism predomination cause that ergonomics researchers can not afford ambiguous solutions and declines the margin of freedom and flexibility in a new concepts' introduction.

To find out how much people are willing to commit to communication and collaboration via any interface, the dimension measuring how people get involved with other life space has to be considered. $49 \%$ of students opted for $(40 \%$ against) explicit, regulated, relations with others, what's more $74 \%$ of respondents would prefer real and personal contact with the boss and that he took into account their personal life. This social profile do not implies interface design as much as the feature how and why users are introduced and related to each other.

The results of the third part of the survey is presented on Fig. 3.

\section{Influence of Technology}

Unfortunately we do not have sufficient technological development rate (66\%) in Poland. This statement suggests that Polish should be treated as novice users. This is important as developing an interface for advanced users should be considerably different from designing for the beginners. What's more the situation is even worse as great amount of us (39\%) are past oriented what means that everything that can occur has took place before and past patterns would be replicated. $40 \%$ of respondents perceive themselves as future oriented, implying expectancy of advancement, improvement and progression. Similarity in tendencies of our society shows our stratification and reveals that Polish become still more and more accustomed to technological innovations. On the other hand interface designers should take into account still great amount of people not familiar with new technologies. These social dimensions may influence navigation process and let the users foresee their perspectives - foresee things that may happen within an interface. This dimension becomes also important when designing "business applications" or "providing historical or visional background or motivation”.

Low technological development rate mentioned above is strongly connected with the extent to which the members of the culture feel threatened by uncertain or unknown situations [14]. Most of people (75\%) perceive new media as uncertain, whereas satisfying the need for certainty will affect the satisfaction the user experiences with the interface. Avoidance of uncertainty cause creation of different values regarding formality, requirements and tolerance for ambiguity. Within interface this factor may highly influence payment possibilities, interaction and navigation design.

Nevertheless answers to subsequent questions revealed that people set their hopes in new technologies (71\%). Among control-oriented cultures technology is perceived as a positive good and mastery can be accomplished through technology [12]. Only $19 \%$ of respondents definitely stated that human beings should live in a harmony with nature and be subjected to the surrounding environment. These opinions show 


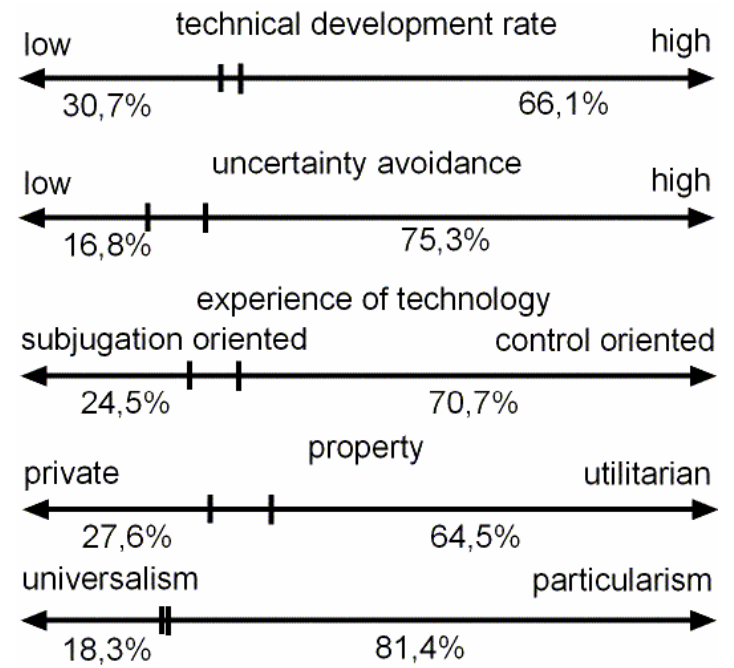

Fig. 4. The results of the last part of the survey.

promising results that Polish are opened for new technologies. This fact is very important as it would be no useful to design the interface if people didn't use it. This factor is critical for the adoption of media as well as the interface. The only problem is suitable interface design, that provides "smart" and "good" technology usage. As far as possible user interface should support user's activities.

The last but disappearing dimension concerns material and intellectual property perception [11]. Almost 65\% of Polish represent utilitarian point of view. They treat land and intellectual achievements as common goods and claim that everyone has to take care of them. This situation seems to be a basis of the whole crisis over intellectual property on the web. It corresponds with particular attitude towards the law. Over $81 \%$ of respondents are able to fail to obey the rights in case of unique circumstances. The degree of adhering to agreed standards of behaviour is considerably law and reveals our contrariness. From one side Polish would like to be appreciated by foreign countries but from the other they do not appreciate foreign achievements.

The exact results of the last part of the survey can be seen on the Fig. 4.

\section{Polish user Interface Profile}

The research revealed wide spectrum of Polish users' preferences. These trends and tendencies should be taken into account during the process of user interface designing. The concluding remarks for interface appearance and functionality can be summed up as follows:

- users do not pay attention to titles

- users do not like reading instructions and take up actions without thinking procedure over. Success can be achieved by clear and understandable interface with suggestive (working) metaphors. Lack of successful interface interaction can discourage user from further interaction.

- information should be presented serendipitously with reasonable but descent amount of options rather then linearly with only one discussed topic. It causes that interface is not boring and attractive for enthusiastic and vehement polish users. At the same time polish users are low contextual and that is why information should be expressed clearly.

- Polish hunger for democracy affects discussion process. Users like to have influence on decision making, take part in discussions and forums. On the other hand they do not put attention to face-saving and as a consequence they do not put confidence to on-line arrangements unless properly certificated. Due to such state only signed and certificated transactions make sense.

- Polish are traditionalists however they pin their hopes on new technologies. People are adaptable and creative but due to minority complex they do not trust domestic products. In the most users' opinion Western Europe or American products are better than Polish. Such an attitude can be changed if Polish find domestic solutions cheaper and more helpful than foreign ones. Polish may eventually accept the solution if it brings benefits. They are usually willing for participating in virtual communities as it is new for them, but the economic point of view become more and more predominating and Polish participants will abandon their membership, if the costs of it are too high.

- Polish are individualists and a group work is very difficult to perform on-line. This ability should be learned at school and developed by means of new media.

- Polish do not expect interface gender diversification, however traditional gender roles disappear and femininity becomes noticeable and female users are sensitive over separate treatment. If the interface is to attract women it should address their needs.

\section{Conclusions}

In the paper we present some indications concerning the process of building user interface, with Polish cultural preferences. The research was done on the basis of different culture dimensions. The results of investigations will allow to design the interface that will fit Polish users profiles requirements and will cause that the information provided on the portal will be accessible, what is the main key to sustainability of virtual learning communities.

Dimensions overview revealed that the interface for Polish users should differ from the one built for other nationalities. Poland is still the developing country where transformations process has begun quite recently. 15 years of an open market existence changed people minds, but unfortunately post-communism bias left considerable amount of old system remnants, what was easily seen during questionnaire responses analysis. Interface design process for Polish users should be based on Polish cultural dimensions profile, even if people preferences still evolve. However most of older persons cannot catch up with prevailing trends the new generation changes .

Polish are used to take patterns from western countries, have minority complex and claim to have a weak political situation. Additionally Polish have low technological 
development rate and they are afraid of uncertain and unpredictable situations. On the other hand still increasing creativity, flexibility and adaptability make the research concerning user interface design very fruitful and interesting.

The research revealed that polish user interface designers should put attention to titles and people positions. They should use active metaphors but unfortunately in a low contextual manner. Information within an interface should be presented serendipitously and there is no need to concentrate on one topic. Polish appeared to be impatient, so progress bars are rather required. Subjects can be presented in an open manner as the discussions are not threatened with moderator integration. People present low acceptance level so any programme faults or instabilities evoke uncertainty. On the other hand lack of face saving dimension and lack of haptic issues cause that users treat electronic mass media in a suspicious way. Confidence can be achieved by means of various certificates, published by foreign, high-authority institution. Polish individualism cause that the system should be designed to let people work individually and subsequent coordination process should be carried out at the final stage.

The interest of Polish people in virtual community membership may be also obtained by providing the benefits of participation. In the case of learning communities they are mostly connected with pedagogic and social aspects. The experience and the investigations showed that all the factors should be taken into account while building and developing the community.

\section{REFERENCES}

[1] H.H. Teo, H.C. Chan, K.K. Wei, Z. Zhang , Evaluating information accessibility and community adaptivity features for sustaining virtual learning communities, International Journal of Human-Computer Studies, vol.59, 2003, 671-697
[2] V.J. Baumgartner, A Practical Set of Cultural Dimensions for Global User-Interface Analysis and Design, master thesis, Wien, 2003

[3] J. Raskin, There is no such thing as information design, in: R. Jacobson (Ed.), Information Design, MIT Press, Cambridge, MA, 1999

[4] M. de Marsico, S. Levialdi, „Evaluating web sites: exploiting user’s expectations,” Int. Journal of Human-Computer Studies, vol.60, 2004, pp. 381-416

[5] P.A. Booth, An Introduction to Human-Computer Interaction, Hove [et. al.]: Erlbaum, 1989

[6] S. McDaniel, What's Your Idea of a Mental Model?, http://www.boxesandarrows.com/archives/print/003253.php

[7] A. Marcus, Dare We Define User-Interface Design?, Interactions vol.9, 2002, pp. 19-24

[8] A. Dix, J. Finlay, G. Abowd, R. Beale, Human-Computer Interaction, New York : Prentice Hall, 1998

[9] A. Wojciechowski, D. Zakrzewska, "Cultural aspects affecting user interface design for virtual learning communities”, in: A. Kieltyk (Ed.), Multimedia in Business \& Education, Foundation of Modern Management (vol. 2), Bialystok, 2005, pp. 136-142.

[10] F. Trompenaars, C. Hampden-Turner, The seven dimensions of culture, http://www.7d-culture.nl/Content/cont042.htm, last visited 8 May 2005

[11] J.C. Condon, F.S. Yousef, An Introduction to Intercultural Communication, Indianapolis: Bobbs-Meril, 1981

[12] D.A. Victor, Cross-Cultural Aspects of International Business Negotiations, http://www.negotiations.com/Demo/ibndemo.pdf, last visited 8 May 2005

[13] E.T. Hall, Beyond culture, New York: Doubleday, 1989

[14] G. Hofstede, Cultures and Organizations: Software of the Mind, London, McGraw-Hill, 1991

[15] A. Marcus and E. W. Gould, "Cultural Dimensions and Global Web User-Interface Design”, ACM Interactions, July/Aug 2000, pp. 32-46.

[16] F.R. Kluckhohn, F.L. Strodtbeck, Variations in value orientations, Evanston: Row, Peterson, 1961

[17] Q. Wright, The Study of International Relations, New York, AppletonCentury-Crofts, 1955

[18] T. Parsons, Talcott Parsons on institutions and social evolutions, selected writings, edited by Leon R. Mayhew, Chicago, University of Chicago Press, 1987

[19] T. Fernandes, Global Interface Design. A Guide to Designing International User-Interfaces, San Diego, Ca. Acad. Press, 1995 